\title{
Ciencia 2.0: catálogo de herramientas e implicaciones para la actividad investigadora
}

\author{
Por Álvaro Cabezas-Clavijo, Daniel Torres-Salinas y Emilio Delgado-López-Cózar
}

\begin{abstract}
Resumen: Se introduce y analiza el concepto de Ciencia 2.0 a través de sus principales propiedades: la participación y la colaboración del usuario así como el libre intercambio de información por medio de aplicaciones web. Se detallan los tipos de aplicaciones que representan las funciones más destacadas de la web 2.0 para investigadores: redes de blogs, revistas con gestión de contenidos 2.0, gestores de referencias online y etiquetado social, open data y reutilización de la información, redes sociales y audio y vídeo-ciencia. Finalmente se discuten los principales factores que inciden en el uso de estas herramientas así como las implicaciones que la adopción generalizada de estos servicios y aplicaciones causarían en la tarea investigadora.
\end{abstract}

Palabras clave: Ciencia 2.0, Web 2.0, Redes sociales, Blogs, Etiquetado social, Gestores online de referencias bibliográficas, Open data, Audio-ciencia, Vídeo-ciencia

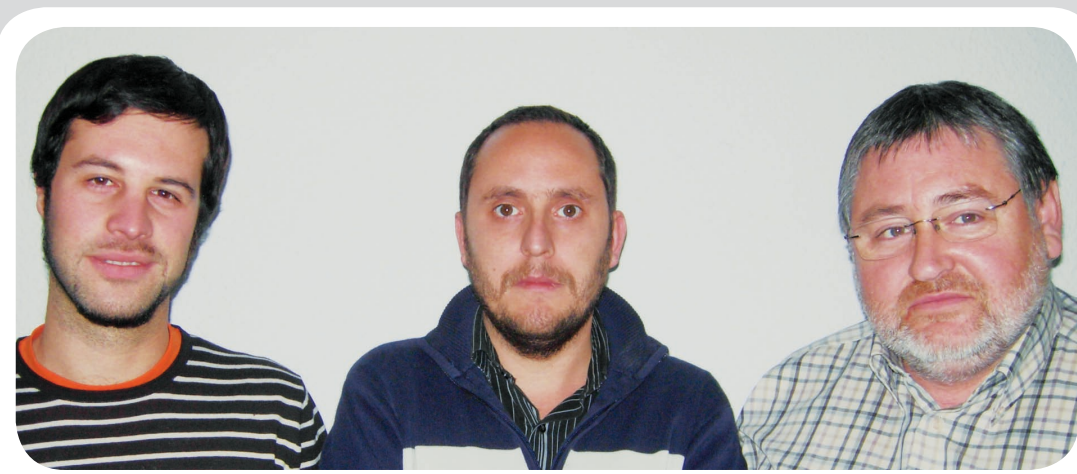

Álvaro Cabezas-Clavijo es licenciado en documentación y máster en información científica por la Universidad de Granada. Miembro del grupo de investigación EC3 (Evaluación de la Ciencia y de la Comunicación Científica) de la misma universidad. Sus principales líneas de investigación son la evaluación bibliométrica de agentes investigadores y el impacto de las herramientas de la web 2.0 en la actividad investigadora.

Daniel Torres-Salinas es doctor en documentación y trabaja como técnico de gestión de la investigación en la Universidad de Navarra, donde realiza auditorías sobre la calidad y el impacto de la investigación. Asimismo es miembro del grupo EC3 (Evaluación de la Ciencia y de la Comunicación Científica) de la Universidad de Granada donde participa en diferentes proyectos y contratos.

Emilio Delgado-López-Cózar es profesor de metodología de la investigación en la Facultad de Comunicación y Documentación de la Universidad de Granada y miembro del grupo EC3. Sus líneas de investigación se centran en la evaluación de las revistas científicas y de la ciencia española, el estudio de la investigación en ByD, y la evaluación del rendimiento científico de instituciones e investigadores.

\section{Title: Science 2.0: Tools catalogue and consequences for scientific activity}

Abstract: The concept of Science 2.0 is introduced and analysed based on its principal characteristics: user participation and collaboration, as well as free information exchange by means of web applications. A categorisation of tools for main web 2.0 functionalities for scientists is detailed: blog networks, journals with 2.0 tools, online reference managers and social tagging, open data and information reutilisation, social networks, and audio and video-science. Main factors influencing the use of these tools are presented. Finally, the consequences for scientific activity of general adoption of these services and applications are discussed.

Keywords: Science 2.0, Web 2.0, Social netwoks, Blogs, Social tagging, Online reference management, Open data, Audioscience, Video-science

Cabezas-Clavijo, Álvaro; Torres-Salinas, Daniel; Delgado-López-Cózar, Emilio. "Ciencia 2.0: catálogo de herramientas e implicaciones para la actividad investigadora". El profesional de la información, 2009, enero-febrero, v. 18, n. 1, pp. 72-79.

DOI: $10.3145 /$ epi.2009.ene.10

\section{Introducción}

LAS APLICACIONES WEB BASADAS EN LA COLABORACIÓN y en el establecimiento de redes sociales por afinidades personales o profesionales han proliferado en los últimos años al calor de la llamada web 2.0.

Plataformas como Facebook, Twitter o Wikipedia se han conver- tido en el referente de un esquema consolidado de internet donde la interacción, la libre publicación de información y la reelaboración constante de contenidos han creado un nuevo paradigma de socialización digital donde, en palabras del creador de Facebook, Mark Zuckerberg, los elementos conectados ya no son páginas sino personas. Compartir fotografías, puntuar con- tribuciones o contar en 140 caracteres qué estás haciendo (Twitter) son sólo algunos ejemplos de sitios basados en la participación y en la formación de comunidades que han alcanzado el éxito en los últimos tiempos.

Pese a la amplia difusión que están teniendo -sólo Wordpress y Facebook sumaron en mayo de 2008 casi 220 millones de visitan- 


\section{"Ciencia 2.0 es el conjunto de servicios y aplicaciones basados en la colaboración y la participación del usuario dentro del campo científico"}

tes únicos (ComScore, 2008)-, la comunidad científica parece haberse quedado al margen de la web 2.0. Un entorno tan eminentemente colaborativo como es la ciencia, construida sobre la base de los descubrimientos y las aportaciones de los pares (colegas homólogos), parece no haber prestado la suficiente atención a estas iniciativas. A pesar de los claros paralelismos que se pudieran establecer entre la ciencia y la web social (Torres-Salinas, 2008), los arraigados mecanismos de control de contenidos han provocado una clara inhibición de los investigadores hacia los entornos abiertos (Mann et al., 2008).

En los últimos años han surgido diversas aplicaciones y plataformas que tratan de ayudar a los científicos en su quehacer diario, ofreciéndoles diferentes herramientas para gestionar sus flujos de trabajo, facilitarles la búsqueda de información pertinente o brindarles nuevos medios para comunicar sus hallazgos. A este movimiento se le ha denominado ciencia 2.0, y se le puede definir, por analogía con la definición de web 2.0, como el conjunto de servicios y aplicaciones basados en la colaboración y la participación del usuario dentro del campo científico. La utilización de este tipo de sistemas podría implicar una rehabilitación extrema de la denominada Hipótesis de Ortega, donde el progreso de la ciencia se basaría en las aportaciones mínimas de una multitud de científicos (Bailón-Moreno et al, 2007). Esta situación, por lo tanto, se vería po- tenciada por diversas aplicaciones y herramientas. Ahora presentamos algunas de las más relevantes.

\section{Redes de blogs científicos}

Las formas de expresión 2.0 más reconocibles y aceptadas por la comunidad científica han sido hasta el momento los blogs, en los que se discuten e intercambian ideas acerca de nuevas investigaciones y corrientes en los distintos campos (Torres-Salinas; Cabezas-Clavijo, 2008). Por blog científico se entiende el sitio web o parte de él, actualizado periódicamente, donde se recopilan por orden cronológico textos o materiales multimedia de uno o varios miembros de la comunidad científica sobre las materias propias de su campo. Sin embargo, las dificultades asociadas a la delimitación conceptual del "blog científico" han provocado que éstos se agrupen en comunidades o redes de blogs que intentan otorgar un sello de calidad y credibilidad a los que se integran en ellas. Entre las más reconocidas encontramos ScienceBlogs (figura 1), una iniciativa comercial surgida en 2006 que aglutina más de 70 blogs y que suma más de un millón de comentarios por parte de sus lectores. En ScienceBlogs caben todo tipo de temas, desde la investigación con células madre a los últimos hallazgos arqueológicos. En España el servicio Madri+d es uno de los más populares en el entorno científico y alberga 70 blogs redactados por expertos. Otra red es la establecida por el grupo editorial Nature, una de las empresas que está dedicando más esfuerzo a implantar una verdadera estrategia 2.0 dentro del mundo de la comunicación científica (Freire, 2007). Nature network, además de un servidor para alojar blogs, pretende servir de punto de encuentro para el debate entre científicos. Otras redes vinculadas a publicaciones científicas, como $B M J$ o PLoS usan los blogs como extensión de sus revistas, ofreciendo opiniones y noticias de actualidad redactadas por editores o empleados del grupo editorial.

\section{http://scienceblogs.com/ http://weblogs.madrimasd.org/ \\ http://network.nature.com/ \\ http://blogs.bmj.com/ \\ http://www.plos.org/cms/blog}

\section{Revistas 2.0}

Más allá de los blogs, el entorno interactivo presenta nuevas posibilidades para las revistas científicas. Una revista 2.0 sería aquella que usa las herramientas de edición y gestión de contenidos propias de la web 2.0, y más concretamente de los blogs, fomentando la participación e interacción entre los actores rela-

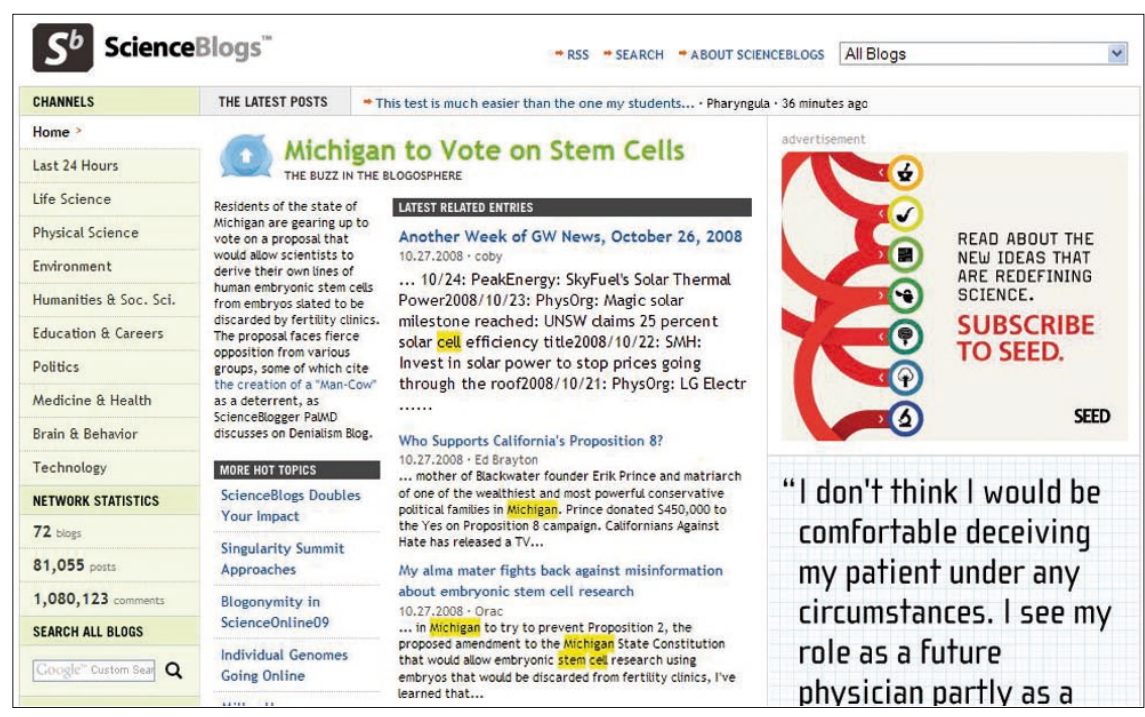

Figura 1: ScienceBlogs 
cionados a ella. Estas herramientas tecnológicas posibilitan asimismo una mejora de los procesos editoriales vinculados a la difusión de la ciencia. El peer review, la imperfecta piedra angular del proceso de certificación científica, ha sido uno de los elementos sobre los que se ha pretendido introducir innovaciones. La revista Nature experimentó con el open peer review, sistema según el cual cualquier miembro registramanuscrito enviado a la revista $\mathrm{Na}$ ture, 2006). Sin embargo, y a pesar de los distintos argumentos en favor de una revisión por pares abierta y transparente (Fernández-Morales; Giménez-Toledo, 2008), la escasa participación de los científicos en la iniciativa de Nature, ampliamente publicitada, hace pensar en la dificultad de motivar a una comunidad científica fuertemente arraigada en sus métodos de trabajo y temerosa de compartir más información de la estrictamente necesaria. Las principales revistas científicas han fomentado este comportamiento penalizando a los autores que adelantan descubrimientos científicos aceptados para su publicación (Elías, 2008).

Otro de los movimientos interesantes relacionados con la publicación científica 2.0 ha sido el promovido por la editorial Public Library of Science (PLoS) que cuenta con siete revistas en acceso abierto. Este editor, nacido en la era digital, do podía valorar la calidad de un

\section{"El sector se encuentra en una fase aún embrionaria y no se vislumbra la aplicación que sirva de punto de encuentro a los científicos"}

se ha convertido en abanderado de la publicación científica 2.0 al incorporar un tipo de edición electrónica que presenta todas las funcionalidades propias de los blogs. La posibilidad de realizar comentarios $\mathrm{y}$ anotaciones, de puntuar los artículos o de recuperar los enlaces emitidos desde la blogosfera son algunas de las particularidades básicas de su revista multidisciplinar PLoS One y, en menor medida, del resto de su catálogo. Asistimos al surgimiento de un entorno híbrido en lo que respecta tanto a la edición electrónica de revistas como a la interacción entre medios. Los nuevos sistemas de gestión de contenidos estimulan los flujos de información entre blogs y revistas científicas. Un ejemplo es el sitio Postgenomic (figura 2), que rastrea el debate que se produce sobre las novedades publicadas en revistas científicas y en la blogosfera. Es posible de este modo comprobar cuáles son las revistas y los blogs más enlazados, así como los artículos y los posts que generan mayor impacto.

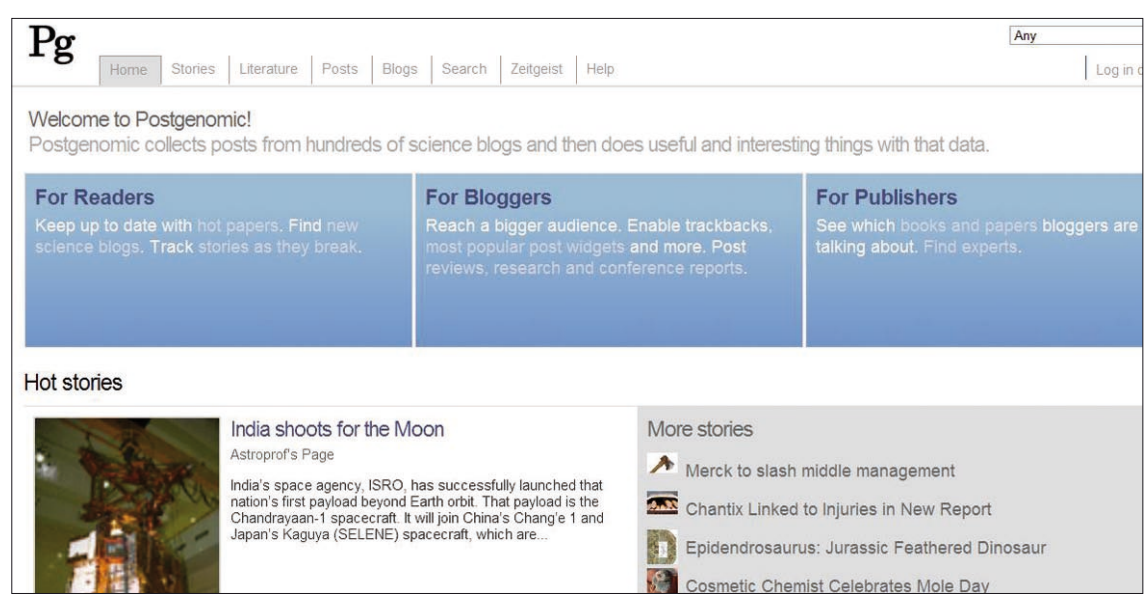

Figura 2: Postgenomic http://www.plos.org/

http://www.postgenomic.com/

\section{Gestores de referencias y sitios de etiquetado social}

Las primeras aplicaciones colaborativas en el campo de la investigación estaban enfocadas a agilizar y enriquecer el proceso de escritura de los artículos científicos, como consecuencia directa de la adaptación de los métodos de producción científica al entorno digital. Así, el software online para el tratamiento de referencias bibliográficas o las extensiones de marcado de favoritos (también denominado etiquetado social) que hacen posible almacenar, clasificar y compartir enlaces en internet han sido algunos de los principales avances en el campo académico 2.0 .

En el caso del software para el tratamiento de referencias bibliográficas cabe destacar el enorme interés de las principales multinacionales de la edición científica por suministrar este tipo de servicios a sus usuarios, normalmente de forma gratuita $y$, por lo general, asociados a sus productos. Un ejemplo es $2 \mathrm{co}$ $\mathrm{llab}$, producto desarrollado por $\mathrm{El}$ sevier y completamente integrado en la base de datos Scopus, de la misma empresa, que ayuda a compartir referencias académicas. Por su parte Nature Publishing Group, siguiendo la estela de sitios como del.ici.ous, lanzó en 2004 Connotea. Según sus creadores su originalidad reside en la combinación de los elementos propios de los gestores de referencias bibliográficas con las nuevas herramientas de etiquetado social (Lund et al, 2005). La misma filosofía subyace en $\mathrm{Ci}$ teULike, producto patrocinado por Springer y con propiedades similares a las aplicaciones previamente señaladas. Por último, señalamos Zotero (figura 3), una extensión de código abierto para el navegador Firefox, que facilita la extracción automática de referencias desde 
sitios web. Otra de sus principales particularidades es su integración con productos ofimáticos así como con gestores de contenidos.

http://www.2collab.com/ http://www.connotea.org/ http://www.citeulike.org/ http://www.zotero.org/

En definitiva, las principales particularidades de estos productos son su interoperabilidad con diferentes bases de datos (importar/exportar referencias), la posibilidad de usarlos desde cualquier ordenador, así como su carácter colaborativo, abierto a la participación de la comunidad científica.

\section{Open data y reutilización de la información}

Dentro del movimiento Open access hay voces que reclaman la publicación de los datos brutos extraídos de las investigaciones financiadas públicamente. De esta forma, en disciplinas como la genética o la bioinformática donde se trabaja con grandes cantidades de datos y donde son necesarias diversas técnicas de data mining para extraer de ellos su significado, la liberación de dichos datos puede significar la oportunidad para que otros grupos de investigación los analicen y realicen hallazgos científicos.

\section{"Los investigadores son reticentes a participar en las aplicaciones $2.0^{\prime \prime}$}

Pero, ¿por qué un investigador, que ha invertido años en conseguir un proyecto de investigación, ha gastado dinero en contratar personal y ha tenido que rellenar diversas solicitudes para la compra de material compartiría los datos de sus investigaciones de forma libre y gratuita con el resto de la comunidad científica? Aunque aparentemente podría interpretarse como

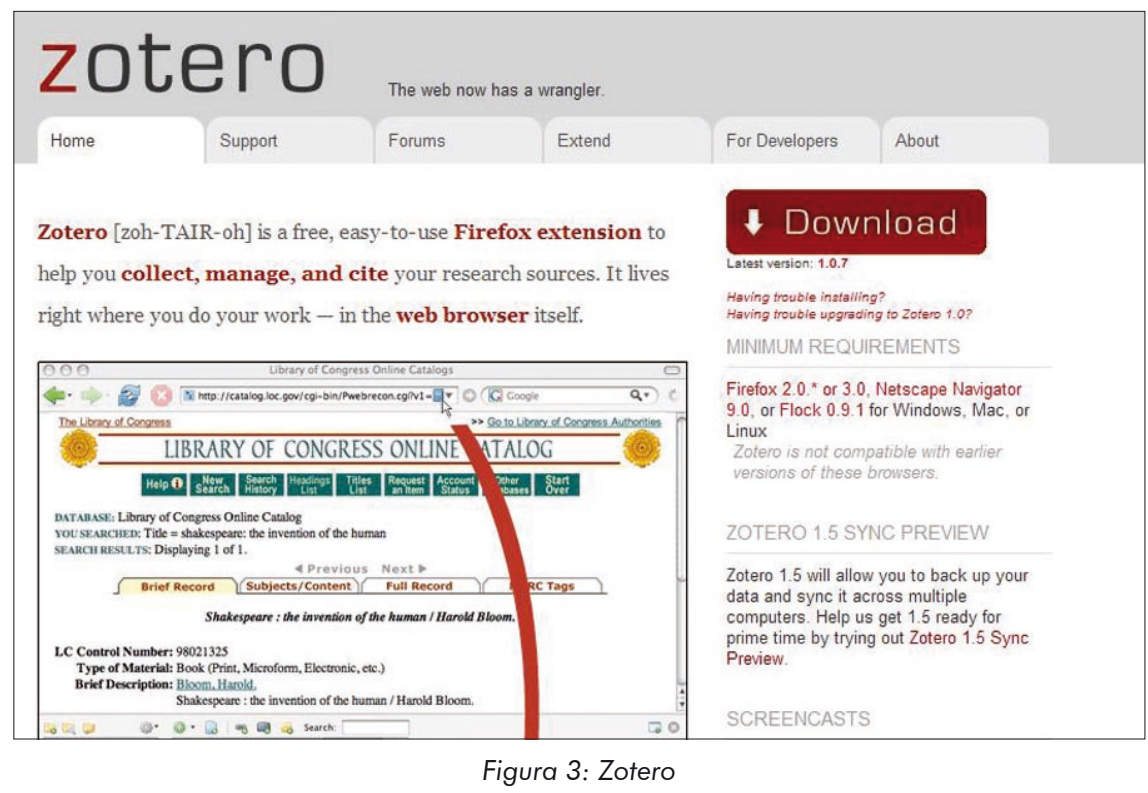

una desinteresada contribución al progreso científico, lo cierto es que las motivaciones para compartir los datos en bruto tomados de un trabajo pueden ir más allá. Así, la puesta a libre disposición de datos puede ser la llave para establecer colaboraciones con otros grupos de investigación, aumentando las posibilidades de generar nuevas publicaciones. Recientes análisis también demuestran que compartir los datos de investigación de forma pública puede incidir positivamente sobre la citación (Piwowar et al, 2007). La acción de compartir datos puede formar parte de una planificación estratégica para aumentar la productividad y el impacto de un grupo investigador.

Son varios los sitios que facilitan compartir datos de investigación con la comunidad científica. Uno de ellos es myExperiment que se define como un entorno virtual de investigación orientado principalmente a la creación de diagramas y representación de flujos de trabajo de experimentos. Aunque es una plataforma orientada a investigadores de ciencias experimentales (los más activos a la hora de compartir sus fuentes de investigación), también puede ser útil para los profesionales de la información, como lo demuestra la presencia de diversas aplicaciones para búsquedas específicas en Medline. Otros sitios en los que se pueden compartir datos, protocolos y todo tipo de materiales de investigación son Labmeeting (figura 4) y OpenWetWare. Estas aplicaciones funcionan como el cuaderno de notas de un laboratorio

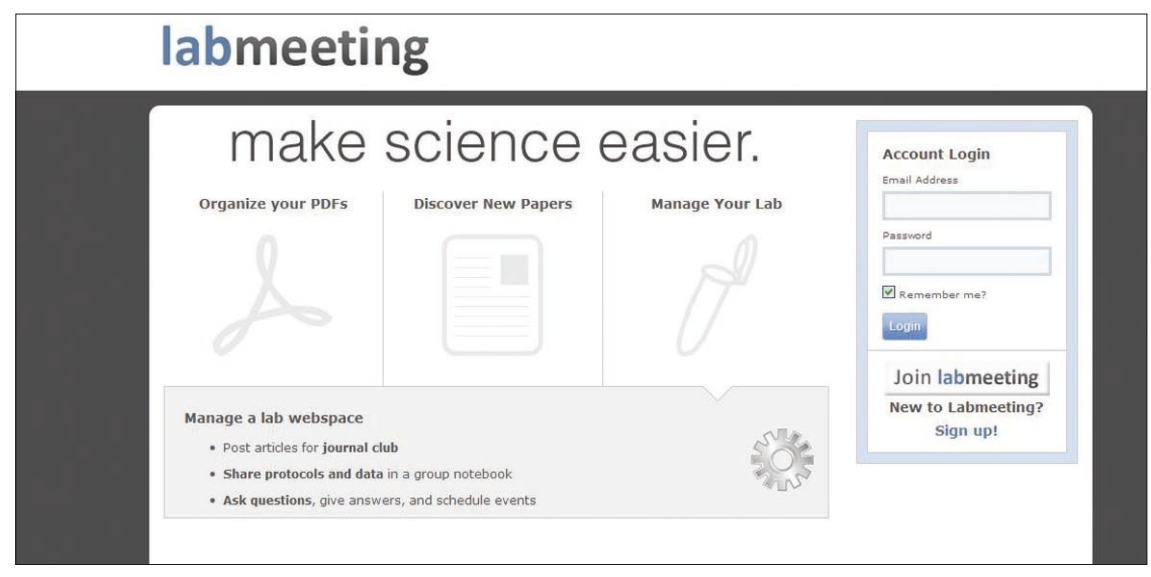

Figura 4: Labmeeting 
y como medio de intercambio de métodos y procesos para el desarrollo de experimentos.

La puesta a disposición pública de conjuntos de datos posibilita su reaprovechamiento y combinación con herramientas de visualización, originando aplicaciones híbridas denominadas mashups. Un mashup es un sitio o aplicación web que usa contenido de otras aplicaciones web para crear un nuevo contenido completo (Wikipedia, 2008). Many eyes, servicio de $I B M$, es una de las más conocidas, y se utiliza para descargar y visualizar datos estadísticos. El proyecto Simile desarrollado por el Massachusetts Institute of Technology (MIT) ofrece también diversas herramientas de código abierto para la reutilización de datos extraídos de otras fuentes permitiendo su visualización en forma de cronologías o bibliografías y facilitando su integración con otras aplicaciones como Zotero.

http://www.myexperiment.org/ http://www.labmeeting.com/ http://openwetware.org/ http://many-eyes.com/ http://simile.mit.edu/

\section{Redes sociales}

Las redes sociales son aplicaciones web donde los usuarios interactúan entre sí y con los materiales multimedia que ellos mismos gene- ran. Dentro del campo científico, uno de los proyectos más interesantes es Mendeley (figura 5) que se define como un sitio social para gestionar, compartir y descubrir referencias bibliográficas. Sus desarrolladores lo consideran el last. fm de los científicos, no en vano el fundador del famoso recommender musical está detrás de su creación. Mendeley tiene como función principal proponer recomendaciones de lectura a los investigadores basándose en los artículos almacenados en su gestor bibliográfico local. Dicho gestor extrae automáticamente los metadatos de la biblioteca de pdfs almacenada en el ordenador del usuario y recopila datos estadísticos acerca de sus lecturas. Esto posibilita la posterior recomendación de nuevas lecturas dentro de su campo de investigación.

Otros sitios como SciLink o Academici proporcionan un amplio espectro de funciones, tales como encontrar investigadores con intereses afines, gestionar lecturas y favoritos, generar redes sociales o subir publicaciones científicas al repositorio. Academia.edu, lanzada a finales del verano de 2008 , es una de las últimas aplicaciones que ha aparecido, y su propósito es poner en contacto a investigadores con los mismos intereses profesionales. El blog SciTechNet(sm) se dedica a documentar de forma sistemática

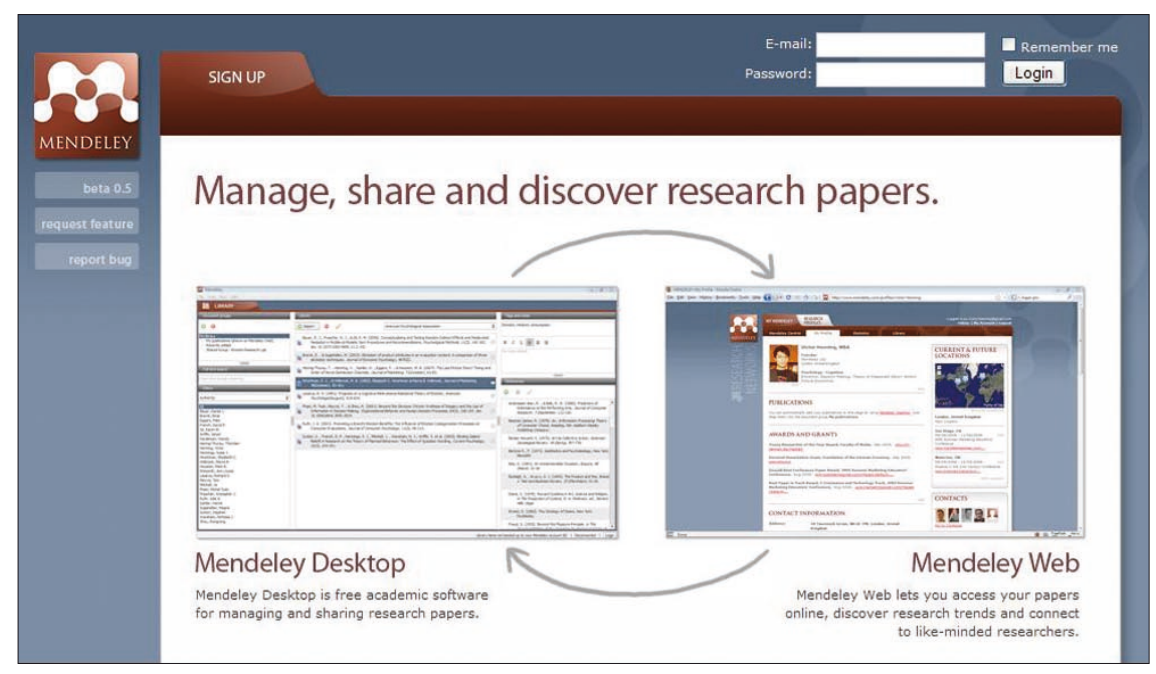

Figura 5: Mendeley las novedades en el campo de las redes sociales científicas.

En cuanto a las redes sociales no debemos dejar atrás Facebook, en la que también hay espacio para la ciencia y donde convergen gran parte de las iniciativas comentadas anteriormente. En primer lugar, mediante la creación de grupos de debate para compartir todo tipo de información. Dentro de estos grupos encontramos revistas científicas (BMJ reader group, El profesional de la información), asociaciones científicas (American Association for the Advancement of Science, $A A A S)$ así como universidades y centros de investigación (Harvard, $M I T)$. No debemos olvidar que $F a$ cebook fue una de las primeras redes sociales de estudiantes universitarios, por lo que tiene una alta orientación hacia el mundo académico e investigador. De hecho, entre los múltiples gadgets disponibles encontramos aplicaciones para gestionar bibliografías (SciBook, Social networking for life scientists), para localizar colegas (Science studies network, Research wizard), realizar búsquedas en bases de datos y bibliotecas (PubMed Search, Worl$d$ Cat) o gestionar datos estadísticos (Socialyze). Todas ellas mantienen el principio de transparencia y son visibles para nuestros contactos a través del News feed.

http://www.mendeley.com/ http://www.scilink.com/ http://www.academici.com/ http://scitechnet.blogspot.com/ http://facebook.com

\section{Audio y vídeo-ciencia}

La comunicación social de la ciencia siempre ha sido una empresa difícil para los científicos, que en muchos casos han considerado esta iniciativa como una vulgarización de su trabajo. Su audiencia principal siempre ha sido la comunidad científica y no la sociedad en general. Sin embargo, algunas de las principales revistas científicas han 
fomentado el uso de clips de audio (podcasts) como una nueva forma de comunicación científica. Nature, $J A M A$ o NEJM publican en formato audio algunas de sus investigaciones más relevantes así como entrevistas con científicos. En España, El profesional de la información publicó dos artículos de su número 17(4) en formato audio, posibilitando la descarga y escucha por parte de los usuarios de su web (El profesional de la información, 2008).

Una de las últimas corrientes en investigación es la retransmisión de proyectos de carácter científico. El canal ResearchChannel, que emite por cable y vía web es una iniciativa de algunas de las más destacadas organizaciones investigadoras en el mundo con el propósito de comunicar la labor de sus científicos. En su web podemos encontrar documentales, entrevistas y conferencias con una finalidad eminentemente divulgativa y pedagógica.

Por su parte, la vídeo-revista Journal of visualized experiments $(J o V E)$ sirve de plataforma para la emisión de experimentos científicos en el campo de la biología. JoVE es a todos los efectos una publicación científica, ya que las contribuciones deben pasar por el proceso de revisión por pares. La principal diferencia es que lo que se publica no es texto, sino vídeo. Su reciente inclusión en la base de datos biomédica más destacada del mundo, Medline, marca un hito en la aceptación por parte de la comunidad científica de estos nuevos métodos de difusión de la práctica científica y abren el camino a la aparición de nuevas publicaciones en formatos no textuales.

Otros sitios como SciVee ofrecen a los científicos la posibilidad de subir vídeos y presentaciones de sus experimentos (pubcasts) permitiendo la discusión entre pares y publicitando sus investigaciones. Algunos casos de análisis expuestos en su web muestran el incremento

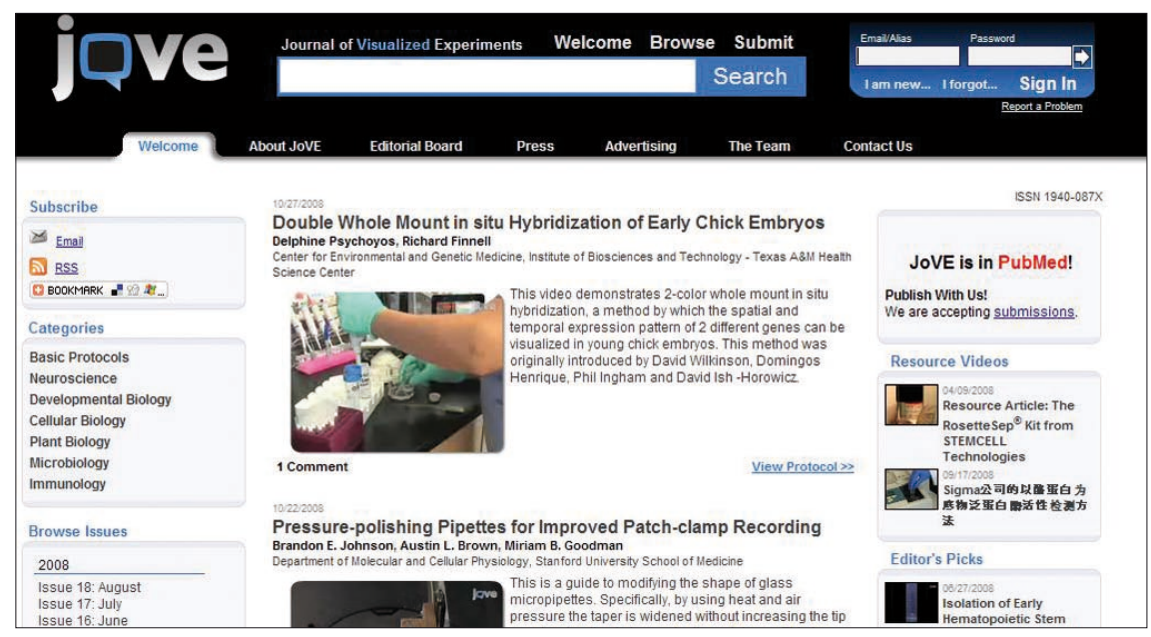

Figura 6: JoVE

de visitas a los artículos vinculados a los pubcasts (SciVee, 2008).

http://www.nature.com/nature/ podcast/

http://jama.ama-assn.org/ authorintheroom/authorindex. $d t l$

\section{http://podcast.nejm.org/}

http://www.researchchannel.org/

http://www.jove.com/

http://www.scivee.tv/

\section{Discusión}

Como hemos visto a lo largo de este trabajo, el abanico de aplicaciones 2.0 al servicio de la comunidad científica es bastante amplio y variado. Sin embargo, el sector se encuentra en una fase embrionaria y todavía no está definida la aplicación que sirva de punto de encuentro a los científicos, del modo que Tuenti o MySpace son puntos de referencia en cuanto a las redes sociales generalistas. Quizás de todos los servicios descritos sea Facebook, nacido en Harvard y extendido a través de las 8 universidades de la Liga Ivy (Brown, Columbia, Cornell, Dartmouth College, Harvard, Univ. de Pensilvania, Princeton y Yale) el que tenga más posibilidades, ya que cuenta con el mayor número de usuarios con estudios universitarios finalizados, el $30 \%$ en el caso de España (Corral, 2008), y por ser una plataforma abierta al resto de las aplicaciones 2.0.
Sin embargo para todas ellas se da una serie de factores críticos que pueden determinar su éxito. El más inmediato y evidente es la participación de los usuarios ya que los servicios de la internet 2.0, y por ende de la ciencia 2.0, necesitan una masa crítica lo suficientemente amplia para resultar útiles.

"Las aplicaciones de la web 2.0 ¿son realmente útiles para los científicos?"

Las reticencias de los investigadores a participar en estas redes se pueden explicar por diversas razones. Juega un papel clave en este contexto la alta competitividad en el trabajo científico. El miedo al robo de ideas o la pérdida de la prioridad en los descubrimientos provoca una discreción en la difusión de resultados que no se rompe hasta que éstos son publicados por medios convencionales. Esta situación se acentúa por el llamado sistema de recompensas ya que los científicos no son evaluados bajo criterios como su participación desinteresada en el entorno social.

A diferencia de las comunidades 2.0, donde el camino hacía la publicación es libre y sin barreras, la gran ciencia surgida tras la II 
Guerra mundial es tutelada y arbitrada por expertos que filtran las contribuciones relevantes. Posteriormente sólo éstas son juzgadas en los procesos de promoción; un método de incentivos que entra en clara contradicción con las prácticas habituales de la web 2.0. Normalmente las aportaciones en un entorno 2.0 (blogs, wikis o preprints) otorgan a su creador la prioridad, pero no la certificación que demanda la comunidad científica y que únicamente se obtiene a través de la publicación en una revista revisada por pares. Como afirma Timo Hannay, jefe de publicaciones web de Nature Publishing Group, la asignación del crédito es una de las grandes barreras para la adopción generalizada de las nociones de la ciencia 2.0 (Waldrop, 2008).

La otra barrera la imponen los mismos métodos de evaluación del trabajo científico; si la participación en la web 2.0 pudiera medirse en términos de calidad y cantidad probablemente no existiría ningún impedimento para su adopción. En este sentido Shneiderman (2008) afirma que, de la misma forma que la capacidad de almacenamiento se mide en peta-bytes, el indicador en la ciencia 2.0 para medir la valía de los científicos, organizaciones o países serán las peta-colaboraciones.

Otro factor que sin duda influirá decisivamente en el éxito de la ciencia 2.0 es la propia edad de los científicos. Según datos de los National Institutes of Health (NIH), la institución financiadora de investigación más grande del mundo, la edad media de los investigadores principales de los proyectos concedidos en 2007 estaba en 51 años (NIH, 2008). Esta cifra se aleja de los estándares de los usuarios actuales de la web 2.0 que, según $R a$ pleaf (2008), se sitúan entre los 14 y los 24 años. Es lógico pensar que la generación que comanda actualmente la ciencia, con unos patrones de uso de internet diferentes a los de las generaciones emergentes, no utilice las aplicaciones 2.0 ya que se apartan de su forma tradicional de comunicación, tanto en el campo personal como en el profesional. Otro de los factores a tener en cuenta es el tiempo de que disponen investigadores y profesores universitarios. Según Simon (2008) un usuario dedicado a generar contenidos destina a estas tareas entre 5-10 horas semanales. Esa cifra aumentará conforme vayamos añadiendo servicios; gestionar una comunidad online como por ejemplo un grupo de Facebook puede aumentar la dedicación a las herramientas 2.0 hasta las 10-20 horas semanales. Sin duda las exigencias del debate científico aumentarían las cifras y pocos investigadores pueden dedicar ese tiempo a dichas labores.

Finalmente, el factor clave que determinará la adopción de estas aplicaciones por parte de los investigadores es su utilidad. ¿Son realmente las herramientas de la web 2.0 útiles para los científicos? Si finalmente estos juzgan que dichas aplicaciones les resultan más eficaces que sus métodos tradicionales, las nuevas generaciones de investigadores interiorizarán estas prácticas y las pondrán en funcionamiento. De lo contrario, la ciencia 2.0 no será más que una nueva etiqueta de marketing al servicio de las grandes editoriales científicas y un reducto de los científicos más extravagantes.

\section{Bibliografía}

Bailón-Moreno, R.; Jurado-Alameda, E.; Ruiz-Banos, R.; Courtial, J. P.; Jiménez-Contreras, E. "The pulsing structure of science: Ortega y Gasset, Saint Matthew, fractality and transfractality". Scientometrics, 2007, v. 71, n. 1, pp. 3-24.

ComScore. Top global web properties.

http://www.comscore.com/press/data/top_ worldwide_properties.asp

Corral, D. "Facebook y Tuenti: la batalla del liderazgo". El país, 1 de octubre de 2008.

http://www.elpais.com/articulo/internet/ Facebook/Tuenti/batalla/liderazgo/elpeputec/ 20081001elpepunet_1/Tes
El profesional de la información. "Escuchar" artículos de El profesional de la información. Zona de Notas, 22 de agosto de 2008.

http://www.elprofesionaldelainformacion.com/ notas $1 \%$ E2\%80\%9Cescuchar\%E2\%80\%9Darticulos-de-el-profesional-de-la-informacion

Elías, C. La razón estrangulada: la crisis de la ciencia en la sociedad contemporánea. Barcelona: Debate, 2008, ISBN 9788483067802.

Fernández-Morales, I.; Giménez-Toledo, E. "La calidad de las revistas científicas y el open peer review". En 3rd International LIS-EPI Meeting 2008. Innovación en información, 24-25 de septiembre de 2008 .

http://www.ciepi.org/lisepi/archivos/material/ fernandezgimenez.pdf

Freire, J. "Estrategia web 2.0 de Nature Publishing Group". En: Juan Freire. Nómada. Reflexiones personales e información sobre la sociedad $y$ el conocimiento abiertos, 14 de octubre de 2007.

http://nomada.blogs.com/jfreire/2007/10/ estrategia-web-.html

Lund, B.; Hammond, T.; Flack, M.; Hannay, T. "Social bookmarking tools (II): A case studyConnotea". D-Lib magazine, 2005, v. 11, n. 4.

Mann, F.; von Walter, B.; Hess, T.; Wigand, R. T. Open access publishing in science: why it is highly appreciated but rarely used. http://openaccesstudy.com/Mann_et_al_2008_ Open_Access_Publishing_in_Science.pdf

Nature. Overview: Nature's peer review trial. 2006.

http://www.nature.com/nature/peerreview/debate/ nature05535.html

NIH. Office of Extramural Research. Average age of principal investigators. 2008.

http://report.nih.gov/NIH_Investment/PDF_ sectionwise/NIH_Extramural_DataBook_PDF/ $N E D B \_S P E C I A L \_T O P I C-A V E R A G E \_A G E . p d f$

Piwowar, H. A.; Day, R. S.; Fridsma, D. B "Sharing detailed research data is associated with increased citation rate". PLoS One, 2007; v. 2, n. 3, e308.

http://www.plosone.org/article/info:doi $\% 2 F 10$. 1371\%2Fjournal.pone.0000308

Rapleaf. Rapleaf study of social network users vs. age. 2008.

http://business.rapleaf.com/company_press_ 2008_06_18.html

SciVee. As an author of a scientific paper, is it worth making a pubcast? 2008. http://www.scivee.tv/worth_it

Shneiderman, B. "Science 2.0". Science, 2008, v. 319, n. 5868 , pp. $1349-1350$.

Simon, N. "How much time does web 2.0 take?". En: Museum 2.0.

http://museumtwo.blogspot.com/2008/04/howmuch-time-does-web-20-take.html

Torres-Salinas, D. "El paradigma 2.0 en las grandes revistas científicas”. En 3rd International LIS-EPI Meeting 2008. Innovación en información, 2008, 24-25 de septiembre de 2008.

http://www.ciepi.org/lisepi/archivos/material/ torres.pdf 
Torres-Salinas, D.; Cabezas-Clavijo, Á. "Los blogs como nuevo medio de comunicación científica". En III Encuentro ibérico de docentes e investigadores en información y documentación 2008, pp. 773-781.

Waldrop, M. M. "Information technologyscience 2.0". Scientific American, 2008, v. 298, n. 5 , pp. $68-73$.

Wikipedia. Marcadores sociales. 2008. http://es.wikipedia.org/w/index.php?title= Marcadores_sociales\&oldid=20989517
Álvaro Cabezas-Clavijo, EC3: Evaluación de la Ciencia y de la Comunicación Científica, Facultad de Comunicación y Documentación, Universidad de Granada. acabezasclavijo@gmail.com

Daniel Torres-Salinas, Centro de Investigación Médica Aplicada.
Universidad de Navarra. torressalinas@gmail.com

Emilio Delgado-López-Cózar, EC3: Evaluación de la Ciencia y de la Comunicación Científica, Facultad de Comunicación y Documentación, Universidad de Granada. edelgado@ugr.es
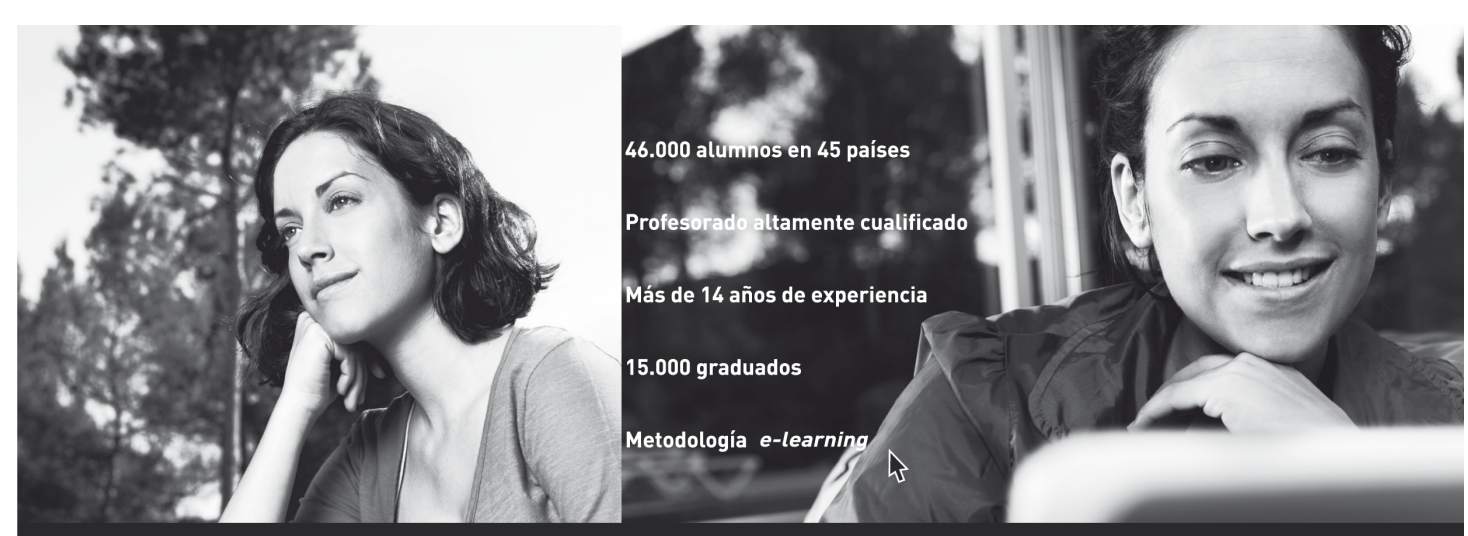

Buscaba un método

personalizado

de estudio

\section{Además encontré una universidad de prestigio}

Por eso elegí la UOC, la primera universidad virtual

\section{ESTUDIOS DE CIENCIAS DE LA INFORMACIÓN Y LA COMUNICACIÓN}

\section{LICENCIATURAS DE $2^{\circ} \mathrm{CICLO}$}

Licenciatura de Documentación

Licenciatura de Publicidad y Relaciones Públicas

Licenciatura de Comunicación Audiovisual

\section{FORMACIÓN DE POSGRADO}

Tecnologías accesibles para los servicios de la sociedad de la información. UOC-Technosite (Máster] Información para la gestión de la innovación (especialización)

Planificación y gestión de servicios y proyectos de información (especialización)

Inicio: febrero 2009. MATRÍCULA VIRTUAL.

Para más información visita nuestra web, www.uoc.edu, llama al 902372373 o acude a cualquiera de nuestras sedes.

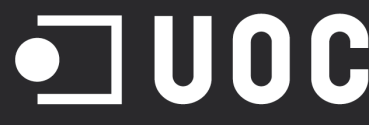

Universitat Oberta de Catalunya

WwW.uoc.edu 


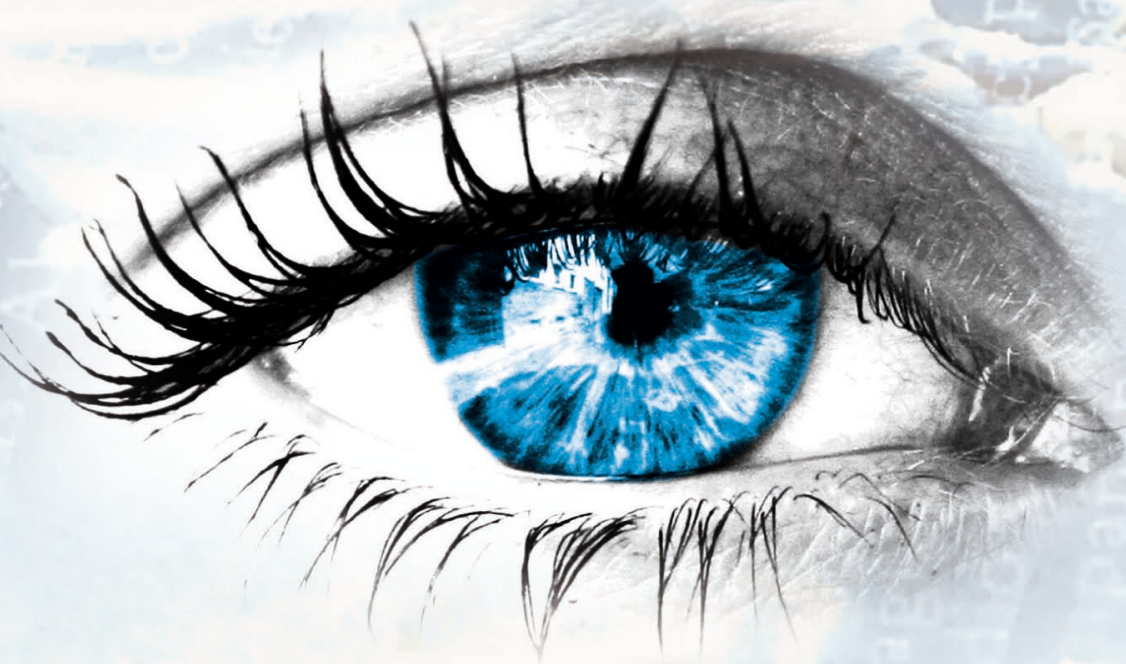

\section{Tenemos otra visión de las bibliotecas.}
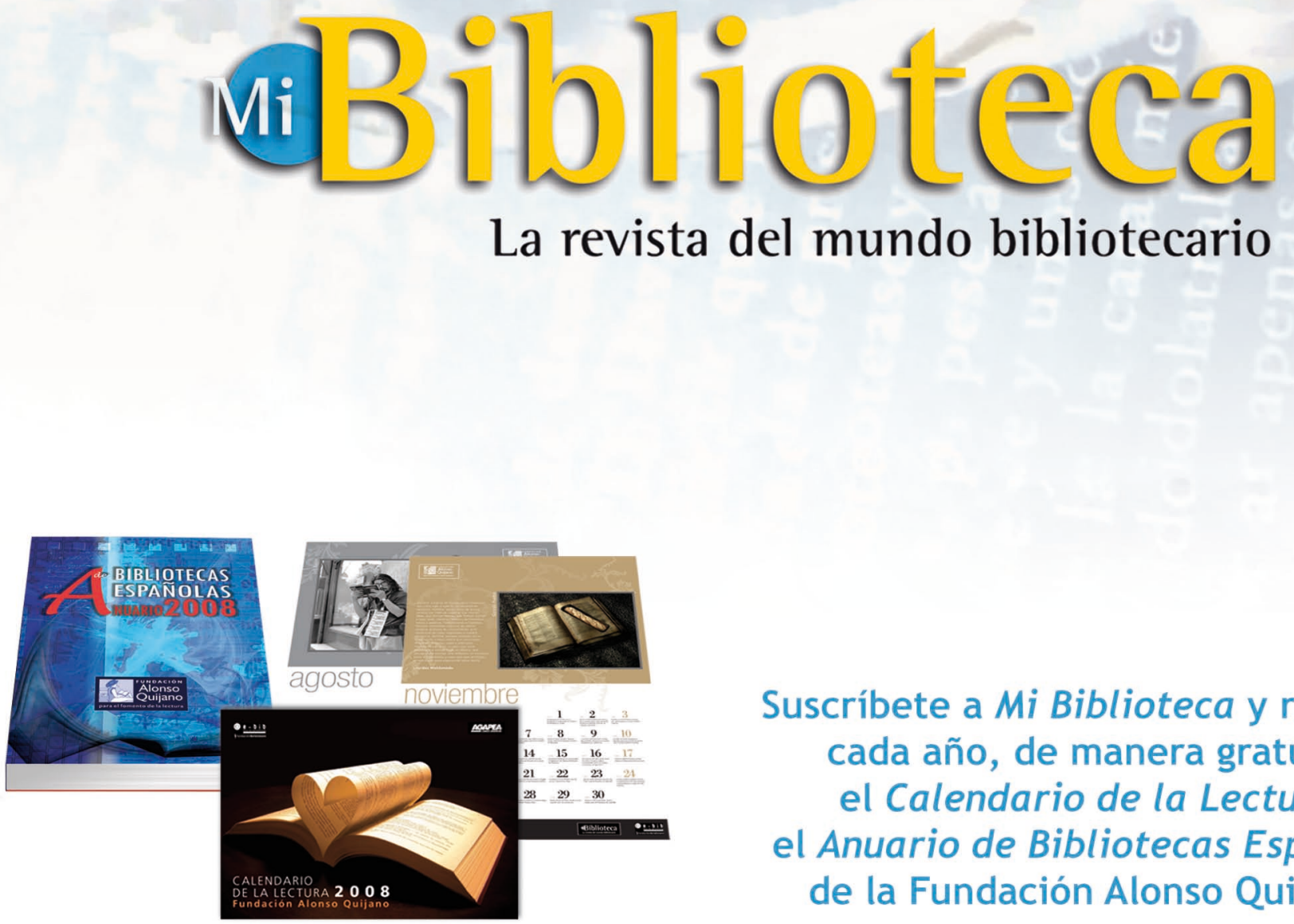

Suscríbete a Mi Biblioteca y recibirás cada año, de manera gratuita, el Calendario de la Lectura y el Anuario de Bibliotecas Españolas de la Fundación Alonso Quijano. 